\section{MS1-P6 From crystal to structure with the versatile macromolecular crystallography beamline I04 at Diamond Light Source}

Ralf Flaig ${ }^{1}$, Jonathan Blakes ${ }^{1}$, Graham Duller ${ }^{1}$, Richard Fearn ${ }^{1}$, Pierpaolo Romano ${ }^{1}$, Irakli Sikharulidze ${ }^{1}$, Graeme Winter ${ }^{1}$, David R. Hall ${ }^{1}$

1. Diamond Light Source, Harwell Science and Innovation Campus, Didcot, OX11 0DE, United Kingdom

email: ralf.flaig@ diamond.ac.uk

Diamond Light Source currently operates five beamlines for macromolecular crystallography (MX) and soon seven beamlines will serve the MX user community. I04, a widely tuneable $(5-25 \mathrm{keV}$, core range $6-14 \mathrm{keV})$ $\mathrm{SAD} / \mathrm{MAD}$ station [1] started with the user programme in early 2007 and is in continuous development in order to improve the experimental capabilities, especially to enable structure solution from increasingly problematic and difficult samples. We have now installed a focusing system using compound refractive lenses allowing the user to change the beam size from $10(\mathrm{~h}) \times 5(\mathrm{v})$ up to 110 (h) $x 100$ (v) microns over a wide energy range and thus be better able to match the beam size to the crystal size or be able to select more precisely the best part of the crystal. To improve signal to noise, beam defining apertures can be used and the data are recorded on a large area pixel array detector. Multi-axis goniometry is used for optimising crystal orientation for phasing experiments, getting more complete and higher multiplicity data or optimizing data collection protocols for radiation sensitive samples. The flexible beamline design also allows for an easy change between standard cryo or room temperature experiments as well as experiments under humidity control and it also enables the beamline to be controlled remotely. We have projects to improve the beamline hardware but are also developing the beamline software further. Data collection strategies (EDNA [2]) for optimised anomalous data collection are provided alongside optimal crystal orientations (XOAlign [3]). GDA [4], the data collection GUI, allows an easy setup of inverse beam or interleaved MAD data sets. Data are automatically processed using various pipelines including FAST_DP [5] and XIA2 [6] and the analysis of the anomalous signal can trigger an automated pipeline (FAST_EP) that provides a quick analysis of the phasing potential of the collected data using no prior information. More complex pipelines are being developed which will use prior information to enable structure solution with the aim to provide crystal to structure capability automatically at the beamline.

\section{References}

[1] http://www.diamond.ac.uk/Beamlines/Mx/I04.html

[2] Incardona, M.-F., Bourenkov, G. P., Levik, K., Pieritz, R. A., Popov, A. N. \& Svensson, O. (2009). J. Synchrotron Rad. 16, 872

[3] https://code.google.com/p/xdsme/

[4] http://www.opengda.org/

[5] https://zenodo.org/record/13039

[6] http://xia2.sourceforge.net

Keywords: Diamond Light Source, macromolecular crystallography, beamlines, experimental phasing, data collection

\author{
MS1-P7 Radiation induced \\ non-isomorphism in protein crystals: a \\ systematic study \\ Gianluca Santoni ${ }^{1}$, Alexander Popov ${ }^{1}$, Gleb Bourenkov ${ }^{2}$
}

1. European Synchrotron Radiation Facility, F38043Grenoble, France

2. EMBL Hamburg Outstation c/o DESY, D-22607 Hamburg, Germany

email: gianluca.santoni@esrf.fr

Increase in the systematic intensity measurements errors under progressing radiation dose is only partly compensated by scaling and radiation-damage corrections (as zero-dose extrapolation [1]). These errors are typically attributed to the variation in the unit cell dimensions and associated small changes in the crystal molecular packing, and to the localized chemical changes known as site-specific radiation damage [2]. We are attempting to understand quantitatively describe these errors on the basis of the structures refined against series of complete data sets collected in massive dose-dependent series. For a number of model systems, specialized data collection protocols were deigned which deliver individual high resolution data sets at low doses of 0.1 to $0.2 \mathrm{MGy}$, ensure sufficient signal-to-noise up to the high doses 10-20 MGy and minimize the influence of other factors as structural inhomogeneity across the crystal volumes or uneven dose deposition. The results indicate that intensity changes are predominantly due to the variation in individual atomic temperature factors. The dependence of isotropic temperature factors on the absorbed dose follows linear relation for most individual atoms, exceptions are the atoms affected by site-specific damage. The slopes of these linear evolutions are distributed according to an inverse gamma function. B-factors variation has been used to determine a mathematical model to describe the decays of intensities and will be presented. [1] Diederichs, K., Mcsweeney, S., \& Ravelli, R. B. G. (2003). Acta Crystallographica Section D Biological Crystallography, 59, 903-909. [2] Garman, E. F. (2010). Acta Crystallographica. Section D, Biological Crystallography, 66, 339-51

Keywords: Radiation damage, non-isomorphism, B-factor 